\title{
Various Psychiatric Manifestation in DiGeorge Syndrome (22q11.2 Deletion Syndrome): A Case Report
}

\author{
Giok Kim², Eunsoo Moon ${ }^{1,2}$, Je Min Park ${ }^{1,2}$, Byung Dae Lee ${ }^{1,2}$, Young Min Lee ${ }^{1,2}$, Hee Jeong Jeong ${ }^{1}$, \\ Soo Yeon Kim ${ }^{1}$, Kangyoon Lee', Hwagyu Suh ${ }^{1}$ \\ ${ }^{1}$ Department of Psychiatry, Medical Research Institute, Pusan National University Hospital, Busan, ${ }^{2}$ Department of Psychiatry, Pusan National \\ University School of Medicine, Yangsan, Korea
}

\begin{abstract}
This case report aimed to describe various psychiatric manifestation and treatment course in a patient with DiGeorge syndrome. Psychiatric symptoms and treatment course in a female patient with DiGeorge syndrome were described. This patient showed psychotic symptoms, mood symptoms, and intellectual disability. As well as various psychiatric symptoms, treatment response and sensitivity of side effect by antipsychotics were different from typical characteristics in psychiatric disorders. This case suggests that the genetic defect in DiGeorge syndrome might have a great association with psychiatric problems and response of antipsychotics.
\end{abstract}

KEY WORDS: DiGeorge syndrome; Psychotic disorders; Bipolar disorder; Depressive disorder; Intellectual disability.

\section{INTRODUCTION}

$22 q 11.2$ deletion syndrome is the most common microdeletion syndrome [1]. These chromosomal abnormalities are often known as $\mathrm{CATCH} 22$ syndrome and include several symptoms such as cardiac anomaly, abnormal face, thymic hypoplasia, cleft palate, and hypocalcemia [1]. Mostly, these chromosomal abnormalities are diagnosed at birth due to a cardiac anomaly [2]. Using fluorescence in situ hybridization (FISH), microdeletion of chromosome 22 can be detected and confirmed [3].

In particular, the relevance between genes in the $22 q 11.2$ region and psychiatric disorders have been studied in several studies [4-6]. These studies have shown that patients with DiGeorge syndrome need to be importantly concerned in the psychiatric area because they can manifest various psychiatric diseases such as autism spectrum disorder (ASD), attention deficit hyperactivity disorder

\footnotetext{
Received: February 20, 2019 / Revised: March 18, 2019

Accepted: March 19, 2019

Address for correspondence: Eunsoo Moon

Department of Psychiatry, Pusan National University Hospital,

Pusan National University School of Medicine, 179 Gudeok-ro,

Seo-gu, Busan 49241, Korea

E-mail: esmun@hanmail.net

ORCID: https://orcid.org/0000-0002-8863-3413
}

(ADHD), intellectual disability, mood and anxiety disorder, and schizophrenia [6,7]. We report a case of patients lately diagnosed as DiGeorge syndrome after diagnosed and treated with psychiatric disorders due to various psychiatric symptoms. This case report was approved by the Pusan National University Hospital Institutional Review Board (approval number, $\mathrm{H}-1901-018-075)$. The informed consent was waived because this was a retrospective study.

\section{CASE}

A 39-year-old female patient was referred to the psychiatric department for psychotic symptoms occurred after treatment of seizure for 25 years. Persecutory delusion, delusion of infidelity, grandiose delusion, and violent behavior occurred after seizure. After psychiatric evaluation, she was diagnosed with "psychotic disorder due to epilepsy', considering that psychotic symptoms were dominant and mostly occurred in the postictal state after frequent seizure attacks at that time.

Her medical history was complex. She suffered from many medical problems, such as epilepsy, diabetes, hypocalcemia, cleft palate and mild intellectual disability

(c) This is an Open-Access article distributed under the terms of the Creative Commons Attribution Non-Commercial License (http://creativecommons.org/licenses/by-nc/4.0) which permits unrestricted non-commercial use, distribution, and reproduction in any medium, provided the original work is properly cited. 
(intelligence quotient [IQ] 65). She had a febrile seizure for three months and a non-febrile seizure for 1 year. She had a cleft palate surgery when she was 7 years old, and she has had a language disturbance since then. She had frequent colds throughout her childhood, which may be caused by an immune dysfunction, and she took a year off at first grade of elementary school. She had low academic achievement due to intellectual disability during elementary school and could not quickly get along with her classmates. When she was 14 years old, a generalized tonic-clonic type of seizure occurred and continued despite of a antiepileptic treatment. Interestingly, the frequency of seizure was lowered after diabetes mellitus and hypocalcemia were treated in the department of endocrinology at the age of 36 . However, she did not take the endocrinological medications regularly, because she did not recognize the necessity of medications due to low intelligence and had no careful familial support.

After frequent relapses of seizure due to the irregular medications, her psychotic symptom occurred at the age of 39. She was diagnosed as 'psychotic disorder due to epilepsy'. After a treatment with antipsychotics, the psychotic symptoms were ameliorated. However, her noncompliance was problematic. After all, her psychotic symptoms deteriorated and consequently admitted to psychiatric hospital for the first time at the age of 41 . However, since then, she showed the course that was different from one of 'psychotic disorder due to epilepsy'. Psychotic symptoms were aggravated irrespective of seizure attack, and sometimes improved at the time of taking no antipsychotics.

Another noticeable aspect is that neuroleptic-induced parkinsonism, such as tremor and rigidity, abruptly occurred at the age of 43 years, even though in regular and consistence dosage of antipsychotics (risperidone, 4 $\mathrm{mg} /$ day) for 2 years. After careful history, she took relatively regular medication before the onset of pseudoparkinsonism. After all, she was admitted to a psychiatric ward due to uncontrolled side effects, all antipsychotics were tapered and changed into conservative medications (benztropine, $2 \mathrm{mg} /$ day; diazepam, $15 \mathrm{mg} /$ day; propranolol, $60 \mathrm{mg} /$ day). After the recovery from pseudoparkinsonism, she went to an outpatient clinic without taking antipsychotics for a year, and psychotic symptoms did not recur in this period.

However, at the age of 44, psychotic symptoms, such as persecutory delusion and auditory hallucination, recurred after all, and she was admitted to a psychiatric ward for the third time. Her clinical course was atypical, contrary to our expectation. One is that psychotic symptoms did not match with the course of epilepsy. The other is that there was hypersensitivity to neuroleptic-induced pseudoparkinsonism even at the same dosage that patient did not previously show pseudoparkinsonism. Therefore, we started to doubt whether current psychiatric diagnosis was correct. In addition, bipolar disorder with psychotic features was considered, because she showed prominent mood symptoms and course fluctuation. However, the period of psychotic symptoms without prominent mood symptoms existed clearly. Schizophrenia was also considered, because psychotic symptoms were independent of seizure attack and psychiatric courses were deteriorating, but the abrupt fluctuation of psychotic symptoms and the change of antipsychotics side effect were not typical manifestation of schizophrenia. Her brain computed tomography showed bilateral basal ganglia calcifications (Fig. 1). Therefore, the probabilities of other medical problems except epilepsy were considered to explain her specific symptom. At last, we considered 22q11.2 deletion syndrome to explain her particular symptoms and performed a FISH test in order to diagnose DiGeorge syndrome. The FISH result showed deletion of 22q11.2 site (Fig. 2).

In summary, psychotic symptoms occurred at age 39 for the first time. Due to the psychotic symptoms were oc-

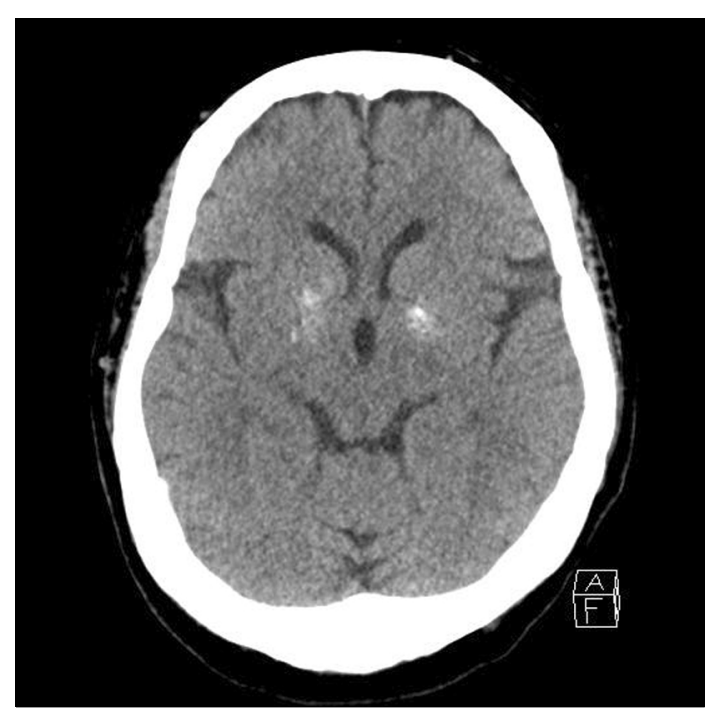

Fig. 1. Bilateral basal ganglia calcifications checked by brain computed tomography scan in a patient with DiGeorge syndrome. 

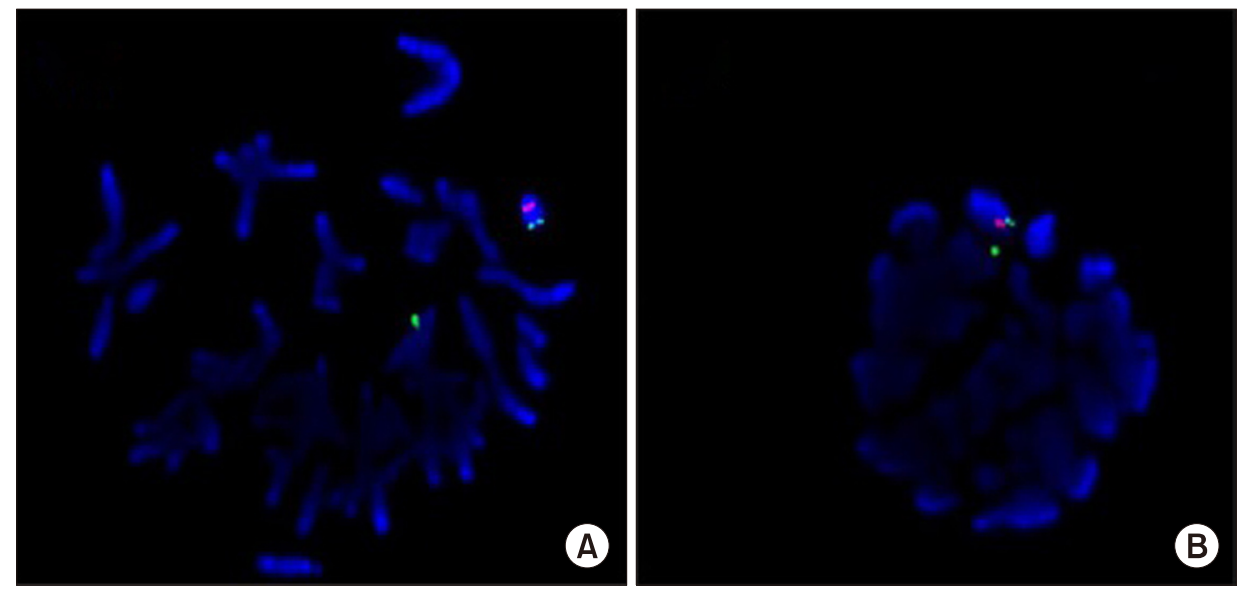

Fig. 2. Results of the fluorescence in situ hybridization (FISH) to confirm 22q11.2 deletion. (A) Metaphase status of the chromosome and (B) interphase status. 22q11.2 region was detected by spectrum pink and the $22 q 13$ region by spectrum green.

curred and exacerbated in the postictal state, she was diagnosed as 'psychotic disorder due to epilepsy' at first. However, psychotic symptoms deteriorated regardless of epilepsy lately and the sensitivity to antipsychotics side effects at the same dosage was fluctuated. Finally, she was diagnosed as DiGeorge syndrome, 22q11.2 deletion syndrome. She showed various psychotic symptoms, manic and depressive symptoms, violent behavior, impulsivity, intermittent anxiety, and intellectual disability.

\section{DISCUSSION}

In most cases, 22q11.2 deletion syndrome is often diagnosed in the neonatal period after birth [8]. However, in this case, several factors prohibited early diagnosis. First, she was delivered at home without prenatal testing. Various assessments about minor malformation were no performed. Second, DiGeorge syndrome was not early diagnosed due to lack of cardiac anomaly in this patient. Third, in perspective of psychiatry, because she was initially similar to postictal psychosis, other diagnoses were not sufficiently considered. Several medical problems and various psychiatric symptoms appeared gradually over time. Various psychiatric symptoms and atypical clinical course leaded to diagnosis of DiGeorge syndrome.

$22 q 11.2$ deletion syndrome is an ultra-high-risk status and is known to be highly susceptible to psychosis $[9,10]$. Several studies have already identified several genes associated with psychosis. This case showed psychotic symptoms, such as persecutory delusion, delusion of infidelity, and grandiose delusion. Psychotic symptoms incidentally occurred when the patient suffered from a recurrent seiz- ure attack. She was similar to postictal and interictal psychosis. One case study reported the relationship between DiGeorge syndrome and interictal psychosis [11]. Our case also showed bilateral basal ganglia calcifications that might be related to recurrent seizures [11]. However, our case showed that the course of psychotic symptoms was different form psychotic disorder due to epilepsy. This patient had various psychiatric symptoms beyond psychotic symptoms. Psychotic symptoms in this patient showed atypical course that could not be fully explained by schizophrenia. As such, neuroleptic-induced parkinsonism occurred even with the same dose of antipsychotics.

There are several genes in $22 \mathrm{q} 11.2$ deletion region, especially COMT gene is related to dopamine regulation [12-14]. 22q 11.2 deletion might lead to COMT haploinsufficiency, that is, the absence of one copy of COMT gene (Met/-, Val/-). Val allele could be related to dopamine dysregulation, behavioral symptomatology and response of antipsychotics treatment $[14,15]$. The defect of COMT gene might explain the occurrence of psychosis and extrapyradmidal symptoms caused by dopamine dysregulation in DiGeorge syndrome [12,14-16]. Furthermore, the expression of COMT gene could be influenced by various factors, such as circadian rhythm and stressful situation $[17,18]$. The combined effects of those factors might have an great influence on dopamine levels in DiGeorge syndrome with the defect of COMT gene. These aspects might be related to the fluctuation of DA level fluctuation and the change of sensitivity to antipsychotics $[19,20]$.

Chromosome 22 is known to accompany other psy- 
chiatry disorders, such as anxiety disorder, mood disorder, ADHD, intellectual disability and autism $[7,8]$. This case showed several symptoms of anxiety disorder and mood disorders, such as elated mood, grandiose delusion, impulsivity, depressed mood, anxiety, and behavioral problems. She also had a mild intellectual disability (IQ 65). However, there was no behavioral problem suspicious of ADHD after careful examination including past personal history and recordings on student life, which describes introvert and passive personality in contrast. The possibility of ASD was also low, because she wanted to have social interaction with a few friends.

In summary, this case showed various psychiatric manifestations that could not fully explained by a single psychiatric disorder. As well as psychiatric symptoms, treatment response and sensitivity of side effects were different from the typical course of psychiatric disorders. Additionally, this patient suffered from various medical diseases. Diagnosis of DiGeorge syndrome was helpful to understand various psychiatric manifestations [8]. Genetic diagnostic approach in patients with various and atypical psychiatric symptoms could be crucial to understand psychiatric symptoms and predict the response of psychotropics in the future.

\section{- Conflicts of Interest}

No potential conflict of interest relevant to this article was reported.

\section{- Author Contributions}

Conceptualization: Je Min Park, Byung Dae Lee, Young Min Lee, Hee Jeong Jeong, Soo Yeon Kim. Data acquisition: Kangyoon Lee, Hwagyu Suh. Formal analysis: None. Funding: None. Supervision: Eunsoo Moon. Writing - original draft: Giok Kim. Writing-review \& editing: Eunsoo Moon.

\section{ORCID}

Giok Kim

Eunsoo Moon

Je Min Park

Byung Dae Lee

Young Min Lee

Hee Jeong Jeong

Soo Yeon Kim

Kangyoon Lee
Hwagyu Suh

https://orcid.org/0000-0002-0360-6708

\section{REFERENCES}

1. Shprintzen RJ, Higgins AM, Antshel K, Fremont W, Roizen N, Kates W. Velo-cardio-facial syndrome. Curr Opin Pediatr 2005; 17:725-730.

2. Ryan AK, Goodship JA, Wilson DI, Philip N, Levy A, Seidel H, et al. Spectrum of clinical features associated with interstitial chromosome 22q11 deletions: a European collaborative study. J Med Genet 1997;34:798-804.

3. McDonald-McGinn DM, Sullivan KE. Chromosome 22q11.2 deletion syndrome (DiGeorge syndrome/velocardiofacial syndrome). Medicine (Baltimore) 2011;90:1-18.

4. Scambler PJ, Kelly D, Lindsay E, Williamson R, Goldberg R, Shprintzen $\mathrm{R}$, et al. Velo-cardio-facial syndrome associated with chromosome 22 deletions encompassing the DiGeorge locus. Lancet 1992;339:1138-1139.

5. Bassett AS, Chow EW. Schizophrenia and 22q11.2 deletion syndrome. Curr Psychiatry Rep 2008;10:148-157.

6. Schneider M, Debbané M, Bassett AS, Chow EW, Fung WL, van den Bree M, et al.; International Consortium on Brain and Behavior in 22q11.2 Deletion Syndrome. Psychiatric disorders from childhood to adulthood in 22q11.2 deletion syndrome: results from the International Consortium on Brain and Behavior in 22q11.2 Deletion Syndrome. AmJ Psychiatry 2014;171:627-639.

7. Cascella M, Muzio MR. Early onset intellectual disability in chromosome 22q11.2 deletion syndrome. Rev Chil Pediatr 2015;86:283-286.

8. Kobrynski LJ, Sullivan KE. Velocardiofacial syndrome, DiGeorge syndrome: the chromosome 22q11.2 deletion syndromes. Lancet 2007;370:1443-1452.

9. Fusar-Poli P, Borgwardt S, Bechdolf A, Addington J, RiecherRössler A, Schultze-Lutter F, et al. The psychosis high-risk state: a comprehensive state-of-the-art review. JAMA Psychiatry 2013;70:107-120.

10. Schultze-Lutter F, Debbané M, Theodoridou A, Wood SJ, Raballo A, Michel C, et al. Revisiting the basic symptom concept: toward translating risk symptoms for psychosis into neurobiological targets. Front Psychiatry 2016;7:9.

11. Tastuzawa Y, Sekikawa K, Suda T, Matsumoto H, Otabe $H$, Nonoyama S, et al. An interictal schizophrenia-like psychosis in an adult patient with 22q11.2 deletion syndrome. Epilepsy Behav Case Rep 2015;3:36-38.

12. Boot E, Booij J, Zinkstok JR, Baas F, Swillen A, Owen MJ, et al. COMT Val(158) met genotype and striatal $D(2 / 3)$ receptor binding in adults with 22q11 deletion syndrome. Synapse 2011;65:967-970.

13. Boot E, Booij J, Zinkstok J, Abeling N, de Haan L, Baas F, et al. Disrupted dopaminergic neurotransmission in 22q11 deletion syndrome. Neuropsychopharmacology 2008;33:1252-1258.

14. Bearden CE, Jawad AF, Lynch DR, Monterossso JR, Sokol S, McDonald-McGinn DM, et al. Effects of COMT genotype on 
behavioral symptomatology in the 22q11.2 deletion syndrome. 2 Deletion Syndrome. Child Neuropsychol 2005;11:109-117.

15. Huang E, Zai CC, Lisoway A, Maciukiewicz M, Felsky D, Tiwari AK, et al. Catechol-O-methyltransferase Val158Met polymorphism and clinical response to antipsychotic treatment in schizophrenia and schizo-affective disorder patients: a meta-analysis. Int J Neuropsychopharmacol 2016; 19:pyv132.

16. Zivković M, Mihaljević-Peles A, Bozina N, Sagud M, NikolacPerkovic M, Vuksan-Cusa B, et al. The association study of polymorphisms in DAT, DRD2, and COMT genes and acute extrapyramidal adverse effects in male schizophrenic patients treated with haloperidol. J Clin Psychopharmacol 2013;33: 593-599.

17. Shirakawa T, Abe M, Oshima S, Mitome M, Oguchi H. Neuronal expression of catechol O-methyltransferase mRNA in neonatal rat suprachiasmatic nucleus. Neuroreport 2004; 15:1239-1243.

18. Armbruster D, Mueller A, Strobel A, Lesch KP, Brocke B, Kirschbaum C. Children under stress - COMT genotype and stressful life events predict cortisol increase in an acute social stress paradigm. Int J Neuropsychopharmacol 2012;15:12291239.

19. Kim SY, Um YH, Lim SC, Jeong JH. Limbic encephalitis manifesting as selective amnesia and seizure-like activity: a case report. Clin Psychopharmacol Neurosci 2018;16:109-113.

20. Aytuluk HG, Simsek T, Yilmaz M, Turan AZ, Saracoglu KT. Can propofol lead to an increase in seizure threshold over the course of electroconvulsive therapy? Clin Psychopharmacol Neurosci 2019;17:523-530. 